\title{
Current Status of Oligometastatic Prostate Cancer: Risk Factors and Treatment Approaches
}

\author{
(D) Fuat Kizilay MD \\ Ege University Faculty of Medicine, Department of Urology, Izmir, Turkey
}

\begin{abstract}
Prostate cancer ( $\mathrm{PCa}$ ) is a common disease that causes significant mortality rates. The widespread use of more sophisticated imaging methods has led to the identification of oligometastatic PCa, which has a limited number of metastases. Local therapy (radical prostatectomy and/or radiotherapy) for the primary tumor and metastasis-directed therapies have been proposed. A number of retrospective analyses have been conducted in this patient group to determine the place of systemic treatment, which is still the recommended standard treatment for metastatic disease. These studies were based on the aims of improving survival, protecting the patient from the potential side effects of systemic therapy, and eliminating local prostate-related symptoms. Although the studies were retrospective in nature, a survival advantage has been demonstrated in patients receiving local treatment or metastasis-directed treatments. It has been also shown in these studies that local treatment has no effect, at least no detrimental effect, on non-oncologic outcomes. However, these studies have significant limitations, primarily their retrospective design, differences in definitions and end-points used, and patient selection biases. Nevertheless, these results are clinically valuable and can be utilized in practice in some special cases. This patient group needs comprehensive standardization and risk stratification. Determining the definition, staging, treatment, and which patients will benefit from local treatment is essential. With ongoing prospective studies, it is expected that these uncertainties will be resolved and there will be revolutionary changes in the treatment of oligometastatic PCa in the near future.
\end{abstract}

Keywords: Metastatic prostate cancer, oligometastatic, radiotherapy, radical prostatectomy, survival

\section{Introduction}

Prostate cancer (PCa) is the second most common cancer in men worldwide with about 1.1 million new cases per year, and almost all patients have metastatic disease at time of PCa-related death (more than 300,000 deaths annually) (1). Current urology guidelines recommend treatment with androgen ablation alone or with chemotherapy as standard treatment for newly diagnosed metastatic PCa (mPCa) (2). There is ample evidence in the literature that $\mathrm{mPCa}$ is highly heterogeneous. Systemic disease may be less aggressive in nature with low metastatic burden, or it may be a highly aggressive form with diffuse metastases $(3,4,5)$. For this reason, although there is no high-level evidence of a survival benefit with primary tumor debulking in oligometastatic $\mathrm{PCa}$ (oligo-mPCa), there is growing interest in surgical treatment for primary tumors in these cases.

Rational for the Treatment of Primary Tumor and Metastases in Oligometastatic Disease

Oligometastatic disease theory was first proposed by Hellman and Weichselbaum (5) in 1995. The authors suggested that progression is a step-by-step process, and that some tumors are at an intermediate level between localized disease and diffuse metastatic disease. This stepwise progression prediction has shown that a group of tumors with low metastatic burden may benefit from local and/or systemic treatment, and some may even 
be cured. The concept of oligometastatic disease and treatment of the primary tumor has been put forward and implemented in colon, lung, breast, and kidney tumors. For example, some colon tumors with a limited number of liver metastases may be cured by surgical treatment of the lesions and adjuvant therapy (6). It is also a well-known fact that cytoreductive nephrectomy in metastatic renal tumors prolongs overall survival and is performed in appropriate patients (7). European Organisation for Research and Treatment of Cancer and the Southwest Oncology Group have shown that nephrectomy prolongs survival by $13-36 \%$ in addition to systemic treatment $(8,9)$. A meta-analysis of 6885 women with advanced-stage ovarian cancer revealed that mean survival was 33.9 months in patient groups with $>75 \%$ maximal cytoreduction and 22.7 months in groups with $<25 \%$ maximal cytoreduction (10).

\section{History and Definition of Oligometastatic Prostate Cancer}

Over the past 20 years, our knowledge of oligometastatic disease has increased (11). Improved imaging modalities and closer follow-up protocols have resulted in a greater number of patients diagnosed with limited metastatic disease $(12,13)$. Many recent genetic and biological studies have shown that primary cancers, limited metastatic cancers, and widespread metastatic cancers actually have different biological behaviors $(14,15,16)$. These findings suggest that not all tumors with limited metastatic lesions will become common metastatic disease, and may have a unique oligometastatic biology. Therefore, distinguishing these tumors from others is very important before planning an aggressive treatment (17).

Definitions of oligo-mPCa have been established based on lesion number by some authors and depending on their location by others. Most of the studies which defined it according to number accepted less than 3, 4, or 5 metastases, and only 1 study accepted less than 10 metastases as oligomPCa $(18,19,20,21)$. The majority of the studies that defined it according to the metastatic location are based on bone or lymph node involvement, but in some prospective studies the classification was made according to extrapelvic involvement $(22,23,24)$. On the other hand, Tabata et al. (25) accepted isolated bone metastases less than half the size of a vertebral body as the metastatic dimension in their definition of oligometastatic disease. The studies defining oligometastatic PCa according to the number, location, and size of the lesions are summarized in Table 1.

Specifically, the effect of a primary tumor-debulking surgery in oligo-mPCa was first described in 2015 by Heidenreich et al. (26). Patients had less than 3 skeletal metastases and prostatespecific antigen (PSA) levels were less than $1.0 \mathrm{ng} / \mathrm{mL}$ after neoadjuvant androgen deprivation therapy (ADT). Patients were divided into 2 groups: those who underwent radical prostatectomy (RP) and those who received only ADT. Median time to castration-resistant PCa was longer in the RP group (40 vs 29 months). In addition, patients treated with RP had longer progression-free (38.6 vs 26.5 months) and cancer-specific survival (CSS) rate (95.6 vs $84.2 \%$ ). The authors concluded that cytoreduction was a feasible and safe treatment method with $39 \%$ overall complication rate and $56.5 \%$ urinary continence recovery rate.
There is a growing interest in local treatment for patients with $\mathrm{mPCa}$, with the hope that local treatment can alter the course of metastatic disease and provide local tumor control, and with the expectation to give the patient the chance of curative treatment by reducing the need for palliative treatment. Patients with mPCa have a median survival of about 3-4 years, and like all oncology patients, deserve more effective treatments that may contribute to their survival (27).

\section{Current Status of Oligometastatic Prostate Cancer Treatment}

There is a large body of data showing the benefits of radical treatment in oligo-mPCa. However, studies in this area are largely retrospective analyses, and ongoing prospective, randomized trials are expected to provide higher quality evidence on this topic. Although the common opinion is that local treatment is feasible in these patients, we are confronted with the fact that the patients in these studies were better candidates for surgical treatment, which is an important factor leading to significant selection bias.

In the majority of these studies, oncologic outcomes such as cancer-specific mortality (CSM) and overall mortality rates and non-oncologic outcomes such as complications, blood loss, and length of hospital stay were evaluated and favorable results achieved. Local treatment in oligo-mPCa patients is an important issue for the surgical treatment of these patients, since the prostate has more local-invasive features and predisposes to more complications. This concern has been the subject of many studies and will also be mentioned below.

Culp et al. (28) first evaluated the oncologic outcomes of local treatment in $\mathrm{MPC}$ a patients. Patients were divided into 3 groups: those who did not receive local treatment, those who underwent RP, and those who received brachytherapy. Overall 5-year survival and predicted CSS rates were significantly higher in the local treatment groups $(\mathrm{p}<0.001)$. In addition, CSM was significantly lower in those who received local treatment $(p<0.01)$. Leyh-Bannurah et al. (29) analyzed CSM in 13,692 patients in the Surveillance, Epidemiology, and End Results Program database. Both radiotherapy (RT) and RP provided lower CSM rates compared to those who did not receive local therapy $(p<0.001)$. In addition, the authors also showed that RP provides lower CSM rates than RT $(p=0.048)$. In a study by Satkunasivam et al. (30), 4069 patients with mPCa were retrospectively analyzed. CSM rate decreased by $52 \%$ with RP and by $62 \%$ with intensity-modulated RT. However, no favorable contribution of conformal RT to oncologic outcomes was observed (30). Another study by Rusthoven et al. (31) in which 538 of 6382 newly diagnosed mPCa patients received prostate RT showed that adding RT to ADT provided an overall survival advantage in both univariate and multivariate analyses. In a second analysis, no significant difference in survival was found between patients with RP added to ADT and patients with RT added to ADT (31). Similarly, Löppenberg et al. (32) classified 15,501 mPCa patients as local treatment and nonlocal treatment groups, with $9.5 \%$ of the patients receiving local treatment. Similar to other studies, they found that 3-year overall survival rates were better in those who received local treatment (69\% vs 54\%, $\mathrm{p}<0.001$ ). 
Although there have been numerous studies showing the benefit of local treatment, all were retrospective series and had selection bias. This limitation is mentioned by Parikh et al. (33), who noted that local treatment was often used in younger ( $<70$ years) patients and patients with fewer comorbidities, lower T stage, lower Gleason score $(<8)$, and lymph nodenegative $\mathrm{mPCa}$. In addition, the authors found that patients who received local treatment had a $50 \%$ lower risk of overall mortality than those who did not.

Oncologic outcomes as well as non-oncologic functional results of local oligo-mPCa treatment, particularly RP, are also a matter of curiosity. In these patients, there is a concern that the prostate may be more invasive and the planned surgical treatment may be more complicated, while on the other hand, there is also an emerging opinion that prostate removal may improve patients' quality of life by preventing local symptoms. Significant complications that may cause adverse consequences in advanced PCa are urinary retention that may require catheterization and transurethral resection, ureteral obstruction, hematuria that may require palliative RT, urinary diversion, and pelvic exenteration. The first single-center, singlesurgeon study for $\mathrm{mPCa}$ patients receiving local treatment in the literature was published by Moschini et al. (34). Although the authors demonstrated favorable complication rates and functional results, they were not able to demonstrate a survival benefit compared to those who did not undergo RP. However, no information was given about the number of metastases in this study (34). In a study by Frazier et al. (35), the rate of symptomatic local progression was $24.6 \%$ in patients diagnosed with node positivity in frozen section analysis after pelvic lymph node dissection who did not undergo RP, and $9.5 \%$ in those who underwent surgery. Moreover, complication rates were $32.6 \%$ and $54.6 \%$, respectively, when local treatment was applied in patients who developed castration-resistant $\mathrm{PCa}$. The results of RP were better than RT (35). The morbidity and mortality associated with local treatment of multimodality therapy in metastatic disease is an important factor for clinicians as well as for patients. The studies evaluating oncologic outcomes of local treatment in oligo-mPCa are summarized in Table 2.

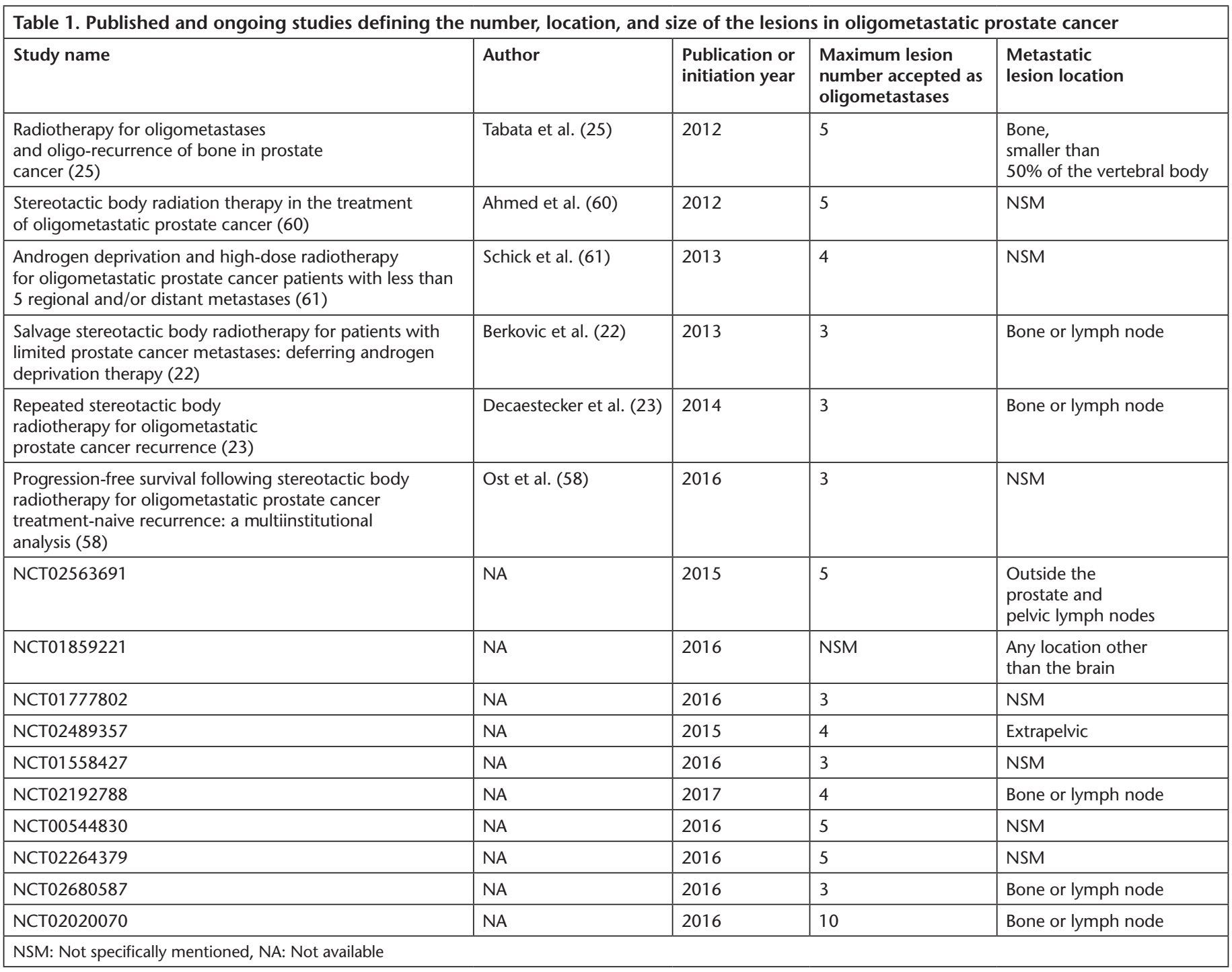


Heidenreich et al. (26) found similar complication rates to those with high-risk localized disease in 23 patients who underwent $\mathrm{RP}$ in metastatic disease. The researchers also emphasized that local progression symptoms developed in $28.9 \%$ of 38 patients who did not undergo surgery, and none of the patients who underwent surgery developed these symptoms (26). Similarly, Sooriakumaran et al. (36) showed that cytoreductive surgery was safe in $106 \mathrm{mPCa}$ patients, with $20.8 \%$ of the patients complication-free after surgery. Steuber et al. (37) compared complication rates in patients with and without RP. Interestingly, the local complication rate was 7\% in the RP-treated group and $35 \%$ in the supportive care group (37). The studies evaluating the functional outcomes of local treatment in $\mathrm{mPCa}$ patients are summarized in Table 3. There are limited data on functional outcomes due to incomplete information in the databases of multicenter studies. It is inevitable that potency rates will be lower in this patient group because none of the MPCa patients are treated with nerve-sparing surgery and these patients may require ADT $(26,36)$. Hormonal therapy may adversely affect postoperative continence rates as well as potency rates (38).

\section{Risk Stratifications That Predict Treatment Success in Oligometastatic Prostate Cancer}

There has been growing interest over the last 20 years in the curing effect of local treatment in oligometastatic disease. The oligometastatic status of these patients should be validated in detail and a stratification system based on clinical and genetic factors is required. First, a definite consensus on the definition of oligometastatic disease should be established, optimal imaging modalities should be identified, clinical and molecular factors predicting disease progression in hormone-sensitive metastatic disease should be identified, and treatment approaches should be optimized. There is a strong need for risk prediction systems, and a risk stratification of these patients is expected with the completion of ongoing prospective clinical trials.

For clinical use, a useful risk stratification system requires molecular classification of genetic, genomic, epigenetic, and microenvironmental factors that affect disease outcomes. An important issue for the definition of oligometastatic disease is the standardization of the imaging methods utilized for diagnosis. The most commonly used imaging modalities for advanced PCa staging are cross-sectional imaging with computed tomography (CT) and magnetic resonance imaging or functional imaging with $99 \mathrm{~m}$-Tc-methylene diphosphonate planar scintigraphy or single-photon emission CT. The low sensitivity of these imaging modalities is an obstacle to their acceptance as a gold standard for routine use in risk classification. Gallium-68-prostate-specific membrane antigen (PSMA)-11 is one of the most sensitive radiotracers and may identify metastatic disease in $54 \%$ of

\begin{tabular}{|c|c|c|c|c|}
\hline Author & Journal, publication year & Intervention & $\begin{array}{l}\text { Accepted oncologic } \\
\text { outcome }\end{array}$ & Result \\
\hline Culp et al. (28) & Eur Urol, 2014 & RP, BT, NLT & 5-year OS and CSS & $\begin{array}{l}\text { Survival rates were higher } \\
\text { in local treatments, in most RP group }\end{array}$ \\
\hline Antwi and Everson (62) & Cancer Epidemiol, 2014 & RP, BT, NLT & OS and CSS & $\begin{array}{l}\text { Survival rates were higher in local } \\
\text { treatments, in most RP group }\end{array}$ \\
\hline Fossati et al. (63) & Eur Urol, 2014 & RP, BT, NLT & CSS and CSM & $\begin{array}{l}\text { LT provided a survival benefit if } \\
\text { predicted 3-year CSM risk }<40 \%\end{array}$ \\
\hline Leyh-Bannurah et al. (29) & Eur Urol, 2017 & $\mathrm{RP}, \mathrm{BT}, \mathrm{NLT}$ & CSM & $\begin{array}{l}\text { LT provided a better CSM ratio, } \\
\text { LT was most beneficial in patients }<1 \text { risk factor }\end{array}$ \\
\hline Rusthoven et al. (31) & JCO, 2016 & RP, NLT & OS and OM & RP provided better OS and OM \\
\hline Löppenberg et al. (32) & Eur Urol, 2016 & $\mathrm{RP}, \mathrm{BT}, \mathrm{NLT}$ & OS and OM & $\begin{array}{l}\text { In the LT group, the survival rate } \\
\text { was higher and the mortality rate was lower }\end{array}$ \\
\hline Parikh et al. (33) & The Prostate, 2017 & RP, IMRT, NLT & OS and OM & $\begin{array}{l}\text { 5-year survival rate favored LT } \\
\text { (RP > IMRT > NLT). The 3-year OM } \\
\text { risk was similar in LT and NLT groups }\end{array}$ \\
\hline Gratzke et al. (54) & Eur Urol, 2014 & RP, NS & OS & 5-year survival rate favored RP \\
\hline Heidenreich et al. (26) & J Urol, 2015 & RP, NLT & OS, CSS, PFS & All 3 factors were in favor of RP \\
\hline Sooriakumaran et al. (36) & Eur Urol, 2015 & $\mathrm{RP}$ & OM & OM rate was $11.3 \%$ \\
\hline Gandaglia et al. (4) & Eur Urol, 2016 & $\mathrm{RP}+\mathrm{ePLND}$ & CSS, PFS & $\begin{array}{l}\text { The 7-year CSS and PFS rates were } \\
82 \% \text { and } 45 \% \text {, respectively }\end{array}$ \\
\hline Steuber et al. (37) & Eur Urol Focus, 2017 & $\mathrm{RP}, \mathrm{BST}$ & OS & No significant difference \\
\hline Bianchini et al. (64) & Clin Genitiurin Cancer, 2017 & LRT, NLT & OS, OM & $\begin{array}{l}\text { Median OS and OM rates were } \\
\text { in favor of LRT }\end{array}$ \\
\hline Cho et al. (65) & PloS One, 2016 & RT, NLT & OS, OM & $\begin{array}{l}\text { 3-year survival rate favored RT, OM } \\
\text { ratio was lower in RT group }\end{array}$ \\
\hline Jang et al. (66) & BJU Int, 2017 & RALP, ADT & CSS, CSM & Both factors were in favor of RALP \\
\hline
\end{tabular}


patients with a PSA value below $1.0 \mathrm{ng} / \mathrm{mL}$, but is currently not widely available (39).

Thompson et al. (40) published the first prospective evidence that treatment of primary $\mathrm{PCa}$ in metastatic disease may reduce mortality. Factors associated with shorter overall survival were widespread disease (appendicular skeletal and/or visceral involvement), bone pain, Gleason score $>8$, and black race. Testosterone at castration level prior to treatment was also associated with poor survival.

Many studies have emphasized the genomic heterogeneity of PCa. It has been shown that every tumor does not respond to systemic treatment in the same way and that genomic differences result in a potentially high- or low-risk phenotype (41). Unpublished data of an ongoing study indicated that a group of metastatic hormone-sensitive mPCa patients with a distinct biological phenotype had a 10-year survival rate of 17\%. In many studies, molecular characterization of primary PCas has been used to assess disease aggressiveness and response rates, but none have specifically evaluated oligometastatic disease $(42,43)$.

Zhao et al. (42) utilized a molecular marker called PAM50, routinely used in breast cancer, in patients undergoing RP. Luminal A subtype had the best prognosis, but only the luminal B subtype benefited from ADT after prostatectomy (42). Nguyen et al. (43) used a molecular marker called Decipher in 235 patients undergoing RP or RT, and reported that biopsy Decipher score was associated with metastatic disease development and PCa-specific mortality. Spratt et al. (44) reported that metastatic risk and PCa-specific mortality could be better predicted by combining the National Comprehensive Cancer Network clinical risk factors and Decipher score. Although none of these genomic tests have been validated in oligometastatic disease, the development of a clinically beneficial risk stratification system is anticipated with the completion of ongoing studies.

\section{Treatment Approaches in Oligometastatic Prostate Cancer}

Whether oligo- or widely metastatic, standard treatment for PCa is long-term palliative ADT with or without chemotherapy. There is a growing body of data about whether primary tumor treatment with stereotactic body RT (SBRT) or RP in oligometastatic disease will improve survival, slow symptomatic disease progression, and reduce the need for palliative surgery $(3,45)$. The 3 main treatment modalities for oligo-mPCa are systemic therapy, primary tumor therapy, and metastasisdirected therapy (MDT).

ADT is still the standard recommended treatment in metastatic disease. Recently, the STAMPEDE, CHARTEED, and GETUG-15 studies have revealed data that support the use of docetaxel with ADT. The outcomes of these studies have shown improved survival and prolonged time to castration-resistant disease (46). However, there is insufficient evidence for a specific recommendation for the oligometastatic patient subgroup. Patients with diffuse metastases have a much higher risk of catastrophic complications such as pathological fractures, spinal compression, and renal insufficiency. Therefore, the application of early ADT in these patients reduces these risks (47). The risk of these complications is much lower in oligometastatic disease. Considering the adverse effects of ADT on morbidity and quality of life, it seems logical to pursue alternative treatments in this group of patients. Despite continuing research for new treatments in oligometastatic patients, docetaxel therapy with ADT is still the standard treatment approach in many urological centers.

It has been shown that cytoreductive or radical surgery to reduce primary tumor burden and RT improve survival in colon, breast, ovarian, and kidney cancers $(10,48,49)$. It has also been shown that radical surgery improves survival in metastatic disease in glioblastoma, renal cell carcinoma, and colorectal cancer $(8,50,51)$. The exact reason underlying these consequences is not fully understood, but the "soil and seed" hypothesis is a logical theory. According to this theory, the tumor cell needs a suitable micro-environment to settle in the metastasis zone. In some studies, it has been shown that primary tumor foci release membrane vesicles, proteins, and nucleic acids that feed the metastatic nest in the locations where circulating tumor cells are located $(52,53)$. In addition, the genetic pathway between primary foci and metastatic foci may also contribute to disease progression. Severing this link by removing the primary tumor may alter the tumor physiology and contribute to the regression or downsizing of metastatic foci.

\begin{tabular}{|l|l|l|l|}
\hline \multicolumn{2}{|l|}{ Table 3. The summary of studies evaluating the functional outcomes of local treatment in metastatic prostate cancer patients } \\
\hline Author & $\begin{array}{l}\text { Journal, } \\
\text { publication year }\end{array}$ & Intervention & Summary of complication comparison \\
\hline Sooriakumaran et al. (36) & Eur Urol, 2015 & RP & Overall complication rate was 20.8\% \\
\hline Steuber et al. (37) & Eur Urol Focus, 2017 & RP, BST & $\begin{array}{l}\text { Severe local complications were 7\% and 35\% } \\
\text { in RP group and BST group, respectively }\end{array}$ \\
\hline Jang et al. (66) & BJU Int, 2017 & RALP, ADT & $\begin{array}{l}\text { There were no urinary tract complications in the RALP group, } \\
\text { while complication rate was between the range 2.4-14.6\% in } \\
\text { the ADT group }\end{array}$ \\
\hline Heidenreich et al. (26) & J Urol, 2015 & RP, NLT & Complication rates were similar in both groups \\
\hline Gandaglia et al. (4) & Eur Urol, 2016 & RP + ePLND & 18\% Clavien 1 and 2, and 0\% Clavien 4 and 5 complications \\
\hline Cho et al. (65) & PloS One, 2016 & RT, NLT & $\begin{array}{l}\text { While no complications were seen in the NLT group, none of } \\
\text { the complications in the RT group were more than grade 3 }\end{array}$ \\
\hline $\begin{array}{l}\text { RT: Radiotherapy, RP: Radical prostatectomy, NLT: No local treatment, ePLND: Extensive pelvic lymph node dissection, BST: Best supportive care, RALP: Robot-assisted } \\
\text { laparoscopic prostatectomy, ADT: Androgen deprivation therapy, PloS: Public Library of Science }\end{array}$ \\
\hline
\end{tabular}


Although there is currently no prospective study showing that primary tumor treatment improves survival in $\mathrm{mPCa}$, there are retrospective studies demonstrating this aforementioned benefit. The Southwest Oncology Group 8894 study in which $1286 \mathrm{mPCa}$ patients were analyzed showed that the risk of death was lower in those who previously underwent RP than those who did not (40). Recent analyses from large databases have shown that 5 -year survival in $\mathrm{MPCa}$ patients treated with radical therapy is higher than those who received systemic treatment alone $(28,54)$. Clinicians are concerned that RP will be more complicated in these patients and will cause more morbidity and mortality. However, this does not seem to be the case. For example, Sooriakumaran et al. (55) showed in a large number of $\mathrm{mPCa}$ patients that cancer-related mortality rate was 3 times higher among those who did not undergo radical treatment compared to those who did. Similarly, in a Swedish study, radical treatment in very high-risk PCa patients was shown to reduce overall mortality (56). In the literature, there is growing evidence that radical or cytoreductive local treatment will contribute to survival in metastatic disease, as long as patients are selected appropriately and the intervention is performed in an adequately experienced center.

The concept of MDT emerged from the concern that metastatic foci could also act as primary foci and lead to other distant metastases. MDT is considered for cases of true oligo-mPCa with a few metastases ( 3 to 5). The main purpose is to control cancer progression, prevent the development of other metastases, and improve quality of life by reducing the need for systemic treatment (5). MDT is routinely recommended in colorectal, sarcoma, and renal cell carcinomas. Studies are usually based on retrospective series and MDT is usually performed in metachronous oligometastases. In a systematic review of 7 studies reporting the outcome of patients receiving MDT for metachronous metastases following primary $\mathrm{PCa}$ treatment, $51 \%$ of patients were progression-free at 1-3 years after MDT (57). In these studies, MDT was performed as surgical metastasectomy or RT. In a study including 119 metachronous oligo-mPCa patients from different centers, a dose-dependent survival advantage with SBRT was detected; higher doses of radiation provided better survival outcomes (58). The results of all these studies indicate that MDT may be a useful treatment modality for metastatic recurrence of $\mathrm{PCa}$ and that the implementation of appropriate local therapies may allow systemic therapy to be delayed in patients with limited metastases (59). However, there is uncertainty regarding the application of these treatments in synchronous metastases. Prospective, randomized trials are likely to answer the question of whether SBRT should be performed alone or in conjunction with RP or RT in synchronous disease. These results are needed before offering these treatments as standard therapy.

\section{Future Expectations and Recommendations Regarding Local Treatments for Oligometastatic Prostate Cancer}

Current data in the literature suggest that local treatments and MDT in MPCa patients are safe and effective. However, using aggressive treatments such as radical surgery in metastatic disease is still a controversial issue. Existing studies are based on retrospective series and only a few studies include an appropriate control group. The design, endpoints, disease definitions, and analysis quality of these studies are highly heterogeneous. Important information such as comorbidities, performance status, baseline PSA information, and the number of metastases that play an important role in survival are missing in some studies. Although a few studies have used propensity score adjustment, the patients treated with local treatment are usually meticulously selected patients and this constitutes an important selection bias.

For now, this treatment modality is in its infancy and a definitive judgment must await the results of ongoing prospective studies. Currently, many randomized controlled trials (e.g. NCT00268476, NCT02454543) are seeking an answer to whether local treatment offers a survival benefit over systemic treatment in $\mathrm{mPCa}$. Currently, aggressive treatment in $\mathrm{mPCa}$ can be recommended only in prospective studies approved by an ethics committee or in the context of a prospective registry of patients having severe morbidity due to the disease. Patients should be informed in detail about the possible benefits and risks of treatment, and their treatment and follow-up should be planned by a multidisciplinary team including a urologist, oncologist, and radiation oncologist.

The optimal treatment of oligo-mPCa will likely become clear in the next few years and clinicians will be able to offer their patients evidence-based therapy. There are advances in diagnostic methods as well as treatment methods; for example, the staging of patients with novel methods such as PSMA-positron emission tomography has emerged, which will influence the number of patients diagnosed with mPCa. Undoubtedly, diagnosis and treatment are 2 closely related modalities and will continue to coevolve.

\section{Conclusion}

Low-volume mPCa is a very heterogeneous disease subgroup within $\mathrm{PCa}$. There is no consensus on the definition, classification, or treatment of the disease. Currently, the widely accepted definition of oligometastatic PCa is the presence of fewer than 5 metastatic lesions that can be detected by imaging modalities. Current data suggest that RP or RT may be safely administered to these patients and may reduce the need for future palliative care. MDTs such as SBRT may also contribute to local cancer control and have low morbidity. At the moment, there is insufficient evidence to make a definitive judgment regarding the impact of aggressive treatments on overall survival or CSS rates. However, at present, the most appropriate approach seems to be the rational use of local treatments as well as systemic treatments after appropriate patient selection and comprehensive clinical evaluation. The genetic and biological characteristics of cancers are also being investigated and this information is expected to contribute to treatment approaches. With the results of prospective, randomized controlled trials, significant changes in disease management are expected in the near future.

\section{Questions}

1. Currently, what is the most commonly accepted definition for oligometastatic prostate cancer? 
2. What is the physiological mechanism of local treatment in oligometastatic prostate cancer and what is the expected benefit from this treatment?

3. What are the recommended treatment options and the recommended indications for oligometastatic prostate cancer?

4. What are the common endpoints of ongoing prospective studies regarding oligometastatic prostate cancer treatment?

\section{Ethics}

Peer-review: Externally peer-reviewed.

Financial Disclosure: The author declared that this study received no financial support.

\section{References}

1. Ferlay I, Soerjomataram I, Dikshit R, et al. Cancer incidence and mortality worldwide: sources, methods and major patterns in GLOBOCAN 2012. Int J Cancer 2015;136:359-386.

2. Morote I, Comas I, Planas J. Re: Nicolas Mottet, Joaquim Bellmunt, Erik Briers, et al. EAU-ESTRO-ESUR-SIOG Guidelines on Prostate Cancer. European Association of Urology; 2017. http://uroweb.org/ guideline/prostate-cancer: How to Assess the Efficacy of Medical Castration. Eur Urol 2018;73:134-135.

3. Ost P, Decaestecker K, Lambert B, et al. Prognostic factors influencing prostate cancer-specific survival in non-castrate patients with metastatic prostate cancer. Prostate 2014;74:297-305.

4. Gandaglia G, Karakiewicz PI, Briganti A, et al. Impact of the Site of Metastases on Survival in Patients with Metastatic Prostate Cancer. Eur Urol 2015;68:325-334.

5. Hellman S, Weichselbaum RR. Oligometastases. I Clin Oncol 1995;13:8-10.

6. Steele G Jr, Bleday R, Mayer RJ, et al. A prospective evaluation of hepatic resection for colorectal carcinoma metastases to the liver: Gastrointestinal Tumor Study Group Protocol 6584. J Clin Oncol 1991;9:1105-1112.

7. Choueiri TK, Xie W, Kollmannsberger C, et al. The impact of cytoreductive nephrectomy on survival of patients with metastatic renal cell carcinoma receiving vascular endothelial growth factor targeted therapy. J Urol 2011;185:60-66.

8. Mickisch GH, Garin A, van Poppel H, et al. Radical nephrectomy plus interferon-alfa-based immunotherapy compared with interferon alfa alone in metastatic renal-cell carcinoma: a randomised trial. Lancet 2001;358:966-970.

9. Flanigan RC, Salmon SE, Blumenstein BA, et al. Nephrectomy followed by interferon alfa- $2 \mathrm{~b}$ compared with interferon alfa- $2 \mathrm{~b}$ alone for metastatic renal-cell cancer. N Engl J Med 2001;345:1655-1659.

10. Bristow RE, Tomacruz RS, Armstrong DK, et al. Survival effect of maximal cytoreductive surgery for advanced ovarian carcinoma during the platinum era: a meta-analysis. J Clin Oncol 2002;20:1248-1259.

11. Reyes DK, Pienta KJ. The biology and treatment of oligometastatic cancer. Oncotarget 2015;6:8491-8524.

12. Khoo V. Is There Another Bite of the Cherry? The Case for Radical Local Therapy for Oligometastatic Disease in Prostate Cancer. Eur Urol 2016;69:13-14

13. Evangelista L, Briganti A, Fanti S, et al. New Clinical Indications for (18) F/(11)C-choline, New Tracers for Positron Emission Tomography and a Promising Hybrid Device for Prostate Cancer Staging: A Systematic Review of the Literature. Eur Urol 2016;70:161-175.

14. Tamoto E, Tada M, Murakawa K, et al. Gene-expression profile changes correlated with tumor progression and lymph node metastasis in esophageal cancer. Clin Cancer Res 2004;10:3629-3638.

15. Wuttig D, Baier B, Fuessel $S$, et al. Gene signatures of pulmonary metastases of renal cell carcinoma reflect the disease-free interval and the number of metastases per patient. Int J Cancer 2009;125:474-482.
16. Lussier YA, Xing HR, Salama JK, et al. MicroRNA expression characterizes oligometastasis(es). PLoS One 2011;6:e28650.

17. Rubin P, Brasacchio R, Katz A. Solitary metastases: illusion versus reality. Semin Radiat Oncol 2006;16:120-130.

18. Vogelstein B, Kinzler KW. The multistep nature of cancer. Trends Genet 1993;9:138-141.

19. Man YG, Gardner WA. Bad seeds produce bad crops: a single stageprocess of prostate tumor invasion. Int J Biol Sci 2008;4:246-258.

20. Heidenreich A, Bastian PJ, Bellmunt J, et al. EAU guidelines on prostate cancer. part 1: screening, diagnosis, and local treatment with curative intent-update 2013. Eur Urol 2014;65:124-137.

21. Sharifi N, Gulley JL, Dahut WL. Androgen deprivation therapy for prostate cancer. JAMA 2005;294:238-244.

22. Berkovic P, De Meerleer G, Delrue L, et al. Salvage stereotactic body radiotherapy for patients with limited prostate cancer metastases: deferring androgen deprivation therapy. Clin Genitourin Cancer 2013; 11:27-32.

23. Decaestecker K, De Meerleer G, Lambert B, et al. Repeated stereotactic body radiotherapy for oligometastatic prostate cancer recurrence. Radiat Oncol 2014;9:135.

24. Tosoian JJ, Gorin MA, Ross AE, et al. Oligometastatic prostate cancer: definitions, clinical outcomes, and treatment considerations. Nat Rev Urol 2017; 14:15-25.

25. Tabata K, Niibe $Y$, Satoh T, et al. Radiotherapy for oligometastases and oligo-recurrence of bone in prostate cancer. Pulm Med 2012;2012:541656.

26. Heidenreich A, Pfister D, Porres D. Cytoreductive radical prostatectomy in patients with prostate cancer and low volume skeletal metastases: results of a feasibility and case-control study. J Urol 2015;193:832-838.

27. Saad F, Fizazi K. Androgen Deprivation Therapy and Secondary Hormone Therapy in the Management of Hormone-sensitive and Castration-resistant Prostate Cancer. Urology 2015;86:852-861.

28. Culp SH, Schellhammer PF, Williams MB. Might men diagnosed with metastatic prostate cancer benefit from definitive treatment of the primary tumor? A SEER-based study. Eur Urol 2014;65:1058-1066.

29. Leyh-Bannurah SR, Gazdovich S, Budäus L, et al. Local Therapy Improves Survival in Metastatic Prostate Cancer. Eur Urol 2017;72:118-124.

30. Satkunasivam R, Kim AE, Desai M, et al. Radical Prostatectomy or External Beam Radiation Therapy vs No Local Therapy for Survival Benefit in Metastatic Prostate Cancer: A SEER-Medicare Analysis. I Urol 2015;194:378-385.

31. Rusthoven CG, Jones BL, Flaig TW, et al. Improved Survival With Prostate Radiation in Addition to Androgen Deprivation Therapy for Men With Newly Diagnosed Metastatic Prostate Cancer. J Clin Oncol 2016;34:2835-2842.

32. Löppenberg B, Dalela D, Karabon $P$, et al. The Impact of Local Treatment on Overall Survival in Patients with Metastatic Prostate Cancer on Diagnosis: A National Cancer Data Base Analysis. Eur Urol 2017;72:14-19.

33. Parikh RR, Byun J, Goyal S, Kim IY. Local Therapy Improves Overall Survival in Patients With Newly Diagnosed Metastatic Prostate Cancer. Prostate 2017;77:559-572.

34. Moschini M, Morlacco A, Kwon E, et al. Treatment of M1a/M1b prostate cancer with or without radical prostatectomy at diagnosis. Prostate Cancer Prostatic Dis 2017;20:117-121.

35. Frazier HA 2nd, Robertson JE, Paulson DF. Does radical prostatectomy in the presence of positive pelvic lymph nodes enhance survival? World J Urol 1994;12:308-312.

36. Sooriakumaran P, Karnes J, Stief C, et al. A Multi-institutional Analysis of Perioperative Outcomes in 106 Men Who Underwent Radical Prostatectomy for Distant Metastatic Prostate Cancer at Presentation. Eur Urol 2016;69:788-794.

37. Steuber T, Berg KD, Røder MA, et al. Does cytoreductive prostatectomy really have an impact on prognosis in prostate cancer patients with 
low-volume bone metastasis? Results from a prospective case-control study. European urology focus 2017.

38. Adam M, Tennstedt $P$, Lanwehr $D$, et al. Functional Outcomes and Quality of Life After Radical Prostatectomy Only Versus a Combination of Prostatectomy with Radiation and Hormonal Therapy. Eur Urol 2017;71:330-336.

39. van Leeuwen PJ, Stricker P, Hruby G, et al. (68) Ga-PSMA has a high detection rate of prostate cancer recurrence outside the prostatic fossa in patients being considered for salvage radiation treatment. Bju Int 2016;117:732-739.

40. Thompson IM, Tangen C, Basler J, Crawford ED. Impact of previous local treatment for prostate cancer on subsequent metastatic disease. J Urol 2002;168:1008-1012.

41. Cancer Genome Atlas Research Network. The Molecular Taxonomy of Primary Prostate Cancer. Cell 2015;163:1011-1025.

42. Zhao SG, Chang SL, Erho N, et al. Associations of Luminal and Basal Subtyping of Prostate Cancer With Prognosis and Response to Androgen Deprivation Therapy. JAMA Oncol 2017;3:1663-1672.

43. Nguyen PL, Haddad Z, Ross AE, et al. Ability of a Genomic Classifier to Predict Metastasis and Prostate Cancer-specific Mortality after Radiation or Surgery based on Needle Biopsy Specimens. Eur Urol 2017;72:845-852.

44. Spratt DE, Dess R, Zhang J, et al. Development and Validation of a Novel Clinical-Genomic Risk Group Classification for Prostate Cancer Incorporating Genomic and Clinicopathologic Risk. Int J Radiation Oncol Biol Phys 2017;99:597.

45. Wiegand LR, Hernandez M, Pisters LL, Spiess PE. Surgical management of lymph-node-positive prostate cancer: improves symptomatic control. Bju Int 2011;107:1238-1242.

46. Vale CL, Burdett S, Rydzewska LHM, et al. Addition of docetaxel or bisphosphonates to standard of care in men with localised or metastatic, hormone-sensitive prostate cancer: a systematic review and meta-analyses of aggregate data. Lancet Oncol 2016;1 7:243-256.

47. [No authors listed]. Immediate versus deferred treatment for advanced prostatic cancer: initial results of the Medical Research Council Trial. The Medical Research Council Prostate Cancer Working Party Investigators Group. Br J Urol 1997;79:235-246.

48. Glehen O, Mohamed F, Gilly FN. Peritoneal carcinomatosis from digestive tract cancer: new management by cytoreductive surgery and intraperitoneal chemohyperthermia. Lancet Oncol 2004;5:219-228.

49. [No authors listed]. Polychemotherapy for early breast cancer: an overview of the randomised trials. Early Breast Cancer Trialists' Collaborative Group. Lancet 1998;352:930-942.

50. Nitta T, Sato K. Prognostic implications of the extent of surgical resection in patients with intracranial malignant gliomas. Cancer 1995; 75:2727-2731.

51. Temple LK, Hsieh L, Wong WD, et al. Use of surgery among elderly patients with stage IV colorectal cancer. I Clin Oncol 2004;22:3475-3484.

52. Costa-Silva B, Aiello NM, Ocean AJ, et al. Pancreatic cancer exosomes initiate pre-metastatic niche formation in the liver. Nat Cell Biol 2015;17:816-826.
53. Peinado H, Alečković M, Lavotshkin S, et al. Melanoma exosomes educate bone marrow progenitor cells toward a pro-metastatic phenotype through MET. Nat Med 2012;18:883-891.

54. Gratzke C, Engel J, Stief CG. Role of radical prostatectomy in metastatic prostate cancer: data from the Munich Cancer Registry. Eur Urol 2014;66:602-603.

55. Sooriakumaran P, Nyberg T, Akre O, et al. Comparative effectiveness of radical prostatectomy and radiotherapy in prostate cancer: observational study of mortality outcomes. BMJ 2014;348:g1502.

56. Stattin P, Sandin F, Thomsen FB, et al. Association of Radical Local Treatment with Mortality in Men with Very High-risk Prostate Cancer: A Semiecologic, Nationwide, Population-based Study. Eur Urol 2017;72:125-134.

57. Ost P, Bossi A, Decaestecker K, et al. Metastasis-directed therapy of regional and distant recurrences after curative treatment of prostate cancer: a systematic review of the literature. Eur Urol 2015;67:852-863.

58. Ost P, Jereczek-Fossa BA, As NV, et al. Progression-free Survival Following Stereotactic Body Radiotherapy for Oligometastatic Prostate Cancer Treatment-naive Recurrence: A Multi-institutional Analysis. Eur Urol 2016;69:9-12.

59. Heidenreich A, Bastian PJ, Bellmunt J, et al. EAU guidelines on prostate cancer. Part II: Treatment of advanced, relapsing, and castrationresistant prostate cancer. Eur Urol 2014;65:467-479.

60. Ahmed KA, Barney BM, Davis BJ, et al. Stereotactic body radiation therapy in the treatment of oligometastatic prostate cancer. Front Oncol 2012;2:215.

61. Schick U, Jorcano $S$, Nouet $P$, et al. Androgen deprivation and highdose radiotherapy for oligometastatic prostate cancer patients with less than five regional and/or distant metastases. Acta Oncol 2013;52:1622-1628.

62. Antwi S, Everson TM. Prognostic impact of definitive local therapy of the primary tumor in men with metastatic prostate cancer at diagnosis: A population-based, propensity score analysis. Cancer Epidemiol 2014;38:435-441.

63. Fossati N, Trinh QD, Sammon J, et al. Identifying optimal candidates for local treatment of the primary tumor among patients diagnosed with metastatic prostate cancer: a SEER-based study. Eur Urol 2015;67:3-6.

64. Bianchini D, Lorente D, Rescigno P, et al. Effect on Overall Survival of Locoregional Treatment in a Cohort of De Novo Metastatic Prostate Cancer Patients: A Single Institution Retrospective Analysis From the Royal Marsden Hospital. Clin Genitourin Cancer 2017;15:801-807.

65. Cho Y, Chang JS, Rha KH, et al. Does Radiotherapy for the Primary Tumor Benefit Prostate Cancer Patients with Distant Metastasis at Initial Diagnosis? PLoS One 2016;11:e0147191.

66. Jang WS, Kim MS, Jeong WS, et al. Does robot-assisted radical prostatectomy benefit patients with prostate cancer and bone oligometastases? Bju Int 2018;121:225-231. 